\title{
Vibrational spectra in glasses
}

\author{
T. S. Grigera ${ }^{1}$, V. Martín-Mayor ${ }^{1}$, G. Parisi ${ }^{1}$ and P. Verrocchio ${ }^{2}$ \\ 1 Dipartimento di Fisica, Università di Roma "La Sapienza", Piazzale Aldo Moro 2, 00185 Roma, Italy \\ INFN sezione di Roma - INFM unità di Roma \\ 2 Dipartimento di Fisica, Università di Trento, Via Sommarive, 14, 38050 Povo, Trento, Italy \\ INFM unità di Trento
}

(November 21, 2018)

\begin{abstract}
The findings of X-ray and neutron scattering experiments on amorphous systems are interpreted within the framework of the theory of Euclidean random matrices. This allows to take into account the topological nature of the disorder, a key ingredient which strongly affects the vibrational spectra of those systems. We present a resummation scheme for a perturbative expansion in the inverse particle density, allowing an accurate analytical computation of the dynamical structure factor within the range of densities encountered in real systems.
\end{abstract}

\section{INTRODUCTION}

Propagating density fluctuation of macroscopic size (hydrodynamic limit) are known to exist both in ordered and in disordered materials. Whereas in the ordered ones those excitations (phonons) persist up to momenta of about one tenth of the Debye momentum, the fate of excitations of microscopical size in disordered systems is still a quite puzzling issue, both from the theoretical and the experimental point of view.

Recently, a lot of experimental attention (Buchenau et al. 1986, Benassi et al. 1996, Foret et al. 1996, Masciovecchio et al. 1996, Masciovecchio et al. 1997, Masciovecchio et al. 1998, Monaco et al. 1998, Sette et al. 1998, Fioretto et al. 1999, Ruocco et al. 1999, Sokolov et al. 1999) has been paid to the high frequency dynamics of disordered systems like glasses. As a matter of fact, high-resolution inelastic X-ray scattering (IXS) and neutron scattering techniques have made accessible to experiment the region where the exchanged external momentum $p$ is comparable to $p_{0}$, namely the momentum where the static structure factor has its first maximum:

$$
0.1 p_{0}<p<p_{0}
$$

The typical momentum $p_{0}$ can be thought as the generalization of the Debye momentum to the case of disordered systems. With these data available for the study of excitations of microscopic size, one is naturally led to ask which of the well-known features of phonons survive even in disordered systems. A number of facts have emerged from the experiments:

1. The dynamic structure factor $S(p, \omega)$ has a Brillouin-like peak for momenta up to $p / p_{0} \sim 0.5$. This inelastic peak is due to the interaction of the external photon (or neutron) with some excitation of the system. A very controversial issue is the propagating nature of the excitations (see, for example, Caponi et al. and Foret et al. in this same volume) within that range of momenta. As a guideline, one could say that as long as the dispersion relation between the position of the peak (in the frequency domain) and the external momentum $\omega_{P}(p)$ is nearly linear, the excitations are likely propagating. Of course, the study of Anderson localization (see, for instance, Parisi 1994) shows that, strictly speaking, this condition is neither necessary nor sufficient, so care should be taken.

2. The peak has a width $\Gamma$, whose dependence on the momentum $p$ within the range (11) has been described by means of the scaling law (for a large variety of materials):

$$
\Gamma \propto p^{\alpha}
$$

Let us remark that, while there seems to be a quite general agreement about the fact that $\Gamma$ is not affected by changes in the temperature, there is still some debate about the actual value of the exponent $\alpha$. More precisely, at low enough momenta (e.g. light scattering) $\alpha$ is undoubtedly $\sim 2$, while at higher momenta (e.g. X-ray scattering) neither the value $\alpha \sim 2$, nor the value $\alpha \sim 4$, describing instead a Rayleigh scattering regime, can be ruled out on experimental grounds.

3. The density of states (DOS) exhibits an excess respect to the Debye behavior $\left(g(\omega) \propto \omega^{2}\right)$, known as the Boson peak. This feature is particularly remarkable for strong glasses. Generally speaking, the typical frequencies of the Boson peak are in the region where the relation between the frequency of the Brillouin-peak $\omega_{p}$ and the momentum $p$ is still linear. 
4. A secondary peak at frequencies smaller than the Brillouin one develops for large momenta, becoming dominant for $p / p_{0} \sim 0.5$.

A number of basic insights on the spectral properties of glasses have been obtained by means of molecular dynamics simulations (Mazzacurati et al. 1996, Dell'Anna et al. 1998, Horbach et al. 1998, Ribeiro et al. 1998, Sampoli et al. 1998, Allend et al. 1999, Feldman et al. 1999, Taraskin and Elliot 1999) on systems such as argon, silica and water. Among the others, let us point out the fact that, in the glass phase, the high frequency dynamics is very well described in the framework of the harmonic approximation. Both dynamic quantities like the dynamic structure factor (Ruocco et al. 2000) and thermodynamic observables as the specific heat (Horbach at al 1999) have been shown to be correctly described by models where only vibrations around quenched positions are taken into account. In figure (11) the numerical results obtained by Ruocco et al. (2000) are shown, which illustrate the above-mentioned spectral features.

On the theoretical side, the study of the short time properties of glasses has been attempted from two different (and somewhat complementary) points of view.

- On one hand, it has been shown (Götze and Mayr 2000) that the Mode Coupling Theory (MCT), which usually describes the long time limit of the time correlators, can be modified in the glassy phase so as to describe only the excitations around the quenched structure. Within such an approach, $S(p, \omega)$ was computed for a hard-sphere glass by means of a generalized hydrodynamics approximation. In this framework, a Brillouin peak with a linear dispersion relation (propagating excitations) is obtained up to $p / p_{0} \sim 0.5$, as well as a secondary peak for higher values. Interestingly, the peak width $\Gamma$ was shown to follow the simple scaling law:

$$
\Gamma_{M C T} \propto p^{2}
$$

only in the very low momenta regime $p / p_{0}<0.1$, whereas a different law applies to the range of momenta comparable with the experimental and simulation values (see the figure (8) in Götze and Mayr 2000) which seems hard to reduce to the simple form (2). Moreover, it has been shown (Ruocco et al. 2000) that, resorting to the harmonic approximation, the memory function can be simply obtained by means of the eigenvalues and eigenvectors of the Hessian matrix.

- A second approach relies on the study of the statistical properties of random matrices (Metha 1991), since within the harmonic approximation the whole dynamical features are encoded in the system's Hessian matrix. A crucial point is to distinguish two different classes of systems:

1. vibrational systems whose disorder has a topological origin, as in glasses (Elliot 1983)

2. vibrational systems on a lattice, where random couplings constants (Schirmacher et al. 1998, Montagna et al. 1999, Kantelhardt et al. 2000, Martin-Mayor et al. 2000, Taraskin et al. 2001) are introduced in order to mimic the behavior of real glasses.

The latter class has recently been studied in some detail (Schirmacher et al. 1998, Taraskin et al. 2001) in order to give an insight into the spectral properties, in particular into the Boson Peak. It turns out (Martin-Mayor et al. 2000), however, that these models definitely miss the $p^{2}$ behavior of the peak width. Because of the long range order due to the underlying lattice, the general beahviour is given instead by

$$
\Gamma_{C P A} \propto p^{4}
$$

even at very low momenta. The above result can be easily obtained within the CPA approximation and can be checked by the direct inspection of the eigenvectors. The discrepancy with the low momenta behaviour of glasses being due precisely to the lack of topological disorder in lattice models, it seems reasonable to address the study of the former class. The problem turns out to reduce to the study of a very special class of random matrices, which have been called Euclidean Random Matrices (ERM) (Mezard et al. 1999). Is is worthwhile to note that this approach would allow to address, besides the study of the high frequency regime of glasses, even the problem of instantaneous normal modes (INM) i.e. the statistical properties of the Hessian matrix of a liquid at equilibrium (Wu and Loring 1992, Wan and Stratt, 1994, Keyes 1997, Biroli and Monasson 1999), the framework being very similar (Cavagna et al. 1999). Nevertheless, in the following we shall focus only on the latter. Recently (Martin-Mayor et al. 2001), the dynamical structure factor $S(p, \omega)$ was analytically computed. The computation relies on a pertubative expansion, the expansion parameter being the inverse density particle $1 / \rho$. Let us recall what those pertubative results undoubtedly have shown: 
- At $\rho \rightarrow \infty$, the dynamical structure factor is composed of a single delta function, whose position changes linearly with the external momentum $p$ at low momenta, representing the undamped propagation of a sound wave in an elastic medium. This can be easily understood by considering the infinite number of particles per wavelength in that limit.

- For high but finite densities, the disorder of the position of the particles involved in the propagation causes a broadening of the Brillouin peak. In other words, the plane waves are no longer eigenstates of the Hessian, hence a finite spreading of the eigenfrequencies involved arises. In principle no analogy with the Rayleigh scattering should be expected. As a matter of fact, the perturbative computation, performed up to $1 / \rho^{2}$ order, shows that at low enough momenta the general behaviour is instead given by:

$$
\Gamma_{E R M} \propto(1 / \rho)^{2} p^{2} .
$$

Yet at density $\rho=1$ a significant deviation from that behaviour is seen as $p / p_{0}$ becomes greater than $\sim 0.1$, in a good qualitative agreement with the results of the MCT approach.

The perturbative calculation has the problem that both the structure factor and DOS end abruptly at a cut-off frequency. Moreover, the experimental densities are of order $\rho \sim 1$ or less (in reduced units), hence the ability of perturbation theory to catch the features of real systems is at least questionable.

In order to overcome those difficulties, we present a resummation scheme for the perturbative results (Grigera et al. 2001) which includes terms at all orders in $1 / \rho$. Within this scheme, a very good agreement with numerical results, even at large frequencies, is found for values of $\rho>0.3$ (reduced units).

\section{HARMONIC APPROXIMATION}

We shall assume that the particles of our system in the glass phase can only oscillate around their equilibrium position, claiming that this is enough to predict the high frequency properties. Here by "equilibrium" we do not mean thermodynamic equilibrium (the thermodynamics of glasses is an entirely different issue than the one addressed here), but rather mechanical equilibrium (i.e. a position where the forces on all particles are zero). For the sake of simplicity, let us consider displacements only along a given direction $\boldsymbol{u}$ :

$$
\boldsymbol{x}_{j}(t)=\boldsymbol{x}_{j}^{\mathrm{eq}}+\boldsymbol{u} \varphi_{j}(t)
$$

Since the number of equilibrium positions $\left\{\boldsymbol{x}_{j}^{\mathrm{eq}}\right\}$ available to the system is actually infinite, growing exponentially with the number of particles $N$, one can ask which one is to take in order to compute the vibrational spectra. The answer lies on the so called self averaging hypothesis, which has proved to be correct for many observables in disordered systems. Hence, we shall assume that in the thermodynamic limit, the spectra obtained for the different realizations of disorder is equal to the one computed by considering the average over the disorder. This statistical approach leads to a tremendous simplification of our task, as shown in the following, because the model is defined simply by the probability distribution of the random variables $\left\{\boldsymbol{x}_{j}^{\mathrm{eq}}\right\}, j=1, \ldots, N$.

Within that harmonic framework, the energy of the system is ( $m$ is the mass of the particles and $\Omega$ is a frequency scale)

$$
V\left(\left\{\varphi_{i}\right\}\right)=\frac{m \Omega^{2}}{2} \sum_{i, j} f\left(\boldsymbol{x}_{i}^{\mathrm{eq}}-\boldsymbol{x}_{j}^{\mathrm{eq}}\right)\left(\varphi_{i}-\varphi_{j}\right)^{2} .
$$

We choose units such that $m=1$ and $\Omega=1$. Thus the Hessian matrix

$$
M_{i j}=\delta_{i j} \sum_{k=1}^{N} f\left(\boldsymbol{x}_{i}^{\mathrm{eq}}-\boldsymbol{x}_{k}^{\mathrm{eq}}\right)-f\left(\boldsymbol{x}_{i}^{\mathrm{eq}}-\boldsymbol{x}_{j}^{\mathrm{eq}}\right)
$$

is an Euclidean random matrix, whose spectral properties we are studying.

In particular, we are interested both in the dynamic structure factor and in the density of states (DOS).

- The dynamic structure factor $S(p, \omega)$, roughly speaking, gives the spectrum of states "excited" by a plane wave with a given momentum $p$. It can be obtained from the Hessian matrix of the potential (7) in the classical limit and in the one excitation approximation (see for instance Martin-Mayor et al. 2001 for a detailed discussion). It reads 


$$
S^{(1)}(p, \omega)=\frac{k_{\mathrm{B}} T p^{2}}{\omega^{2}} \overline{\sum_{n}|\langle n \mid \boldsymbol{p}\rangle|^{2} \delta\left(\omega-\omega_{n}\right)}
$$

where $|n\rangle$ are the eigenvectors of the Hessian matrix (8), the eigenfrequencies $\omega_{n}$ are the square root of the eigenvalues (which are all positive, see equation(7) $), T$ is the temperature, the overline stands for the average on the equilibrium positions $\left\{\boldsymbol{x}_{j}^{\text {eq }}\right\}$, while $|\boldsymbol{p}\rangle$ stands for a momentum plane wave $\left(\langle j \mid \boldsymbol{p}\rangle=\exp \left[\mathrm{i} \boldsymbol{p} \cdot \boldsymbol{x}_{j}^{e q}\right] / \sqrt{N}\right.$, where $|j\rangle$ represents the vector where the $j$-th particle has displacement $\boldsymbol{u}$ and the other particles do not move).

- The DOS describes instead the density of 'all' the vibrational states existing within the system. Interestingly, at the level of one-excitation approximation, the DOS can be obtained by:

$$
g(\omega)=\frac{\omega^{2}}{k_{\mathrm{B}} T p^{2}} S^{(1)}(p \rightarrow \infty, \omega)
$$

The above theoretical result has been pointed out only very recently (Martin-Mayor et al. 2001). Although it can be checked very easily by means of numerical simulations, we believe it would be also very interesting to check it on real systems, using the experimental data at large momenta which are now available. On the other hand, at very high frequencies the one-excitation approximation does not hold, and many-excitations contributions should be taken into account, hence the relation (10) is obviously only an approximation, whose reliability in describing real systems is a very interesting matter. Moreover, we shall see in the following that (10) is crucial in obtaining a model independent derivation of the actual small $p$ behaviour of the width of the Brillouin peak, holding only for topologically disordered systems. As a matter of fact, the same argument cannot be applied to lattice models, because the relation (10) is not true in topologically ordered models.

As mentioned, the Hessian is averaged over the disorder, i.e. over all the allowed equilibrium positions. Those are clearly distributed in a highly correlated manner, due to the hard-core repulsion and long range attraction of the potential. It was shown (Martin-Mayor et al. 2001) that, at the level of the superposition approximation, the correlations can be taken into account by doing an uncorrelated average while at the same time suitably renormalizing the interaction $f$ : if the $k$ point static correlation function is written as

$$
g\left(r_{1}, \ldots r_{k}\right)=g\left(r_{1}\right) \ldots g\left(r_{k}\right),
$$

then the inverse-density expansion for the dynamic structure factor of a system with correlation function $g(r)$ and force $F(r)$ can be obtained from the expansion of a fully uncorrelated system defining a dressed interaction:

$$
f(r) \equiv g(r) F(r)
$$

where the value of the "spring" constant $F(r)$ is weighted by the probability to find two centers of oscillations at the relative distance $r$.

\section{NON PERTURBATIVE RESULTS}

Let $\hat{f}(p)$ be the Fourier transform of $f(r)$. The main 'object' to compute is the resolvent of the Hessian matrix (8), which depends on the complex variable $z=\omega^{2}+i \eta$. It can be written in the following way:

$$
\begin{aligned}
G(p, z) & =\frac{1}{z-\epsilon(p)-\Sigma(p, z)}, \\
\epsilon(p) & =\rho[\hat{f}(0)-\hat{f}(p)] .
\end{aligned}
$$

where complex self-energy $\Sigma(p, z)$ has been introduced. Exploiting the well known relation between resolvent and dynamical structure factor (letting aside the prefactor $k_{\mathrm{B}} T p^{2}$, unessential for the $\omega$ dependence):

$$
S^{(1)}(p, \omega)=-\frac{1}{\omega \pi} \lim _{\eta \rightarrow 0} \operatorname{Im} G\left(p, \omega^{2}+i \eta\right) .
$$

the following connections between the main features of the dynamical structure factor and the self-energy are established: 
- The 'bare' dispersion relation $\epsilon(p)$, which would give the position of the peak in the elastic medium limit, is renormalized by the real part of the self-energy $\Sigma^{\prime}(p, z)$. This gives $\omega^{\text {renorm }}(p)$, the position of the maximum of the structure factor in the frequency domain. Let us note that $\omega^{\text {renorm }}(p)$ is certainly linear for small $p$, as expected.

- The imaginary part $\Sigma^{\prime \prime}(p, z)$ computed at the position of the peak $\omega=\omega^{\text {renorm }}(p)$ gives the width, $\Gamma(p)$, of the $S^{(1)}(p, \omega)$ by means of:

$$
\Sigma^{\prime \prime}\left(p, \omega^{\text {renorm }}(p)\right)=\omega^{\text {renorm }}(p) \Gamma(p)
$$

The self-energy can be obtained as a series in $1 / \rho$ : the $k^{\text {th }}$-order corresponds to $k$ particle-label repetitions when calculating the moments of the $S^{(1)}(p, \omega)$ (the details can be found in Martin-Mayor et al. 2001). It is easy to show that the sum of all the cactus diagrams (see figure (21)) is given by the solution of the following integral equation:

$$
\Sigma(p, z)=\frac{1}{\rho} \int \frac{d^{D} q}{(2 \pi)^{D}} \frac{[\rho(\hat{f}(\boldsymbol{q})-\hat{f}(\boldsymbol{p}-\boldsymbol{q}))]^{2}}{z-\epsilon(q)-\Sigma(q, z)} .
$$

Interestingly, Equation(17) provides a model-independent derivation of the $p^{2}$ scaling of width of the peak of the $S^{(1)}(p, \omega)$. Indeed, the large $q$ contribution to the imaginary part of the integral in equation (17) is, because of (10)

$$
\Sigma_{\infty}^{\prime \prime}(p, z)=-\pi \rho g_{\lambda}(\lambda) \int \frac{d^{D} q}{(2 \pi)^{D}}(\hat{f}(\boldsymbol{q})-\hat{f}(\boldsymbol{p}-\boldsymbol{q}))^{2} .
$$

where $g_{\lambda}(\lambda)$ is the density of states in the domain of eigenvalues $\left(\lambda=\omega^{2}, g_{\lambda}\left(\omega^{2}\right)=\frac{g(\omega)}{2 \omega}\right)$. If the spectrum is Debye-like we have $g_{\lambda}(\lambda) \propto \lambda^{0.5}$, and it is straightforward to show that (18) is proportional to $\omega^{\text {renorm }}(p) p^{2}$. Then the relation (16) implies the scaling:

$$
\Gamma(p) \propto p^{2}
$$

irrespective of the function $f(r)$. Clearly this is only the large $q$ contribution to the integral, but it has been shown (Grigera et al. 2001) that it indeed controls the peak width at small $p$.

\section{THE GAUSSIAN CASE}

In the following we shall study the simple case

$$
f(r)=\exp \left[-r^{2} /\left(2 \sigma^{2}\right)\right] .
$$

This may seem an oversimplification, too distant from any realistic case. However, it is not actually the case, at least for small momenta. For the sake of comparison we can see in figure (3) the Fourier transform of the function $g(r) F(r)$ for Argon at very low temperature $(\sim 10 K)$ together with that of $(20)$. We thus expect our results to be reasonable for momenta smaller than the first zero of the Fourier transform of $g(r) F(r)$, which in general is close to the maximum of the static structure factor, $p_{0}$. Since the Fourier transform of our force decreases an order of magnitude by $p_{0}=2 / \sigma$, we shall take this as our $p_{0}$ during the following discussion, and $\sigma$ will be our unit of length.

We have numerically solved equation (17) to find the self-energy for several values of $\rho$ with the Gaussian choice (20), thus obtaining the structure factor and the density of states, in the eigenvalue domain. In figure (4) we show $S^{(1)}(p, \lambda)$ for several values of the momentum as obtained from equation (17) for $\rho=1$. We also plot the structure factor obtained numerically by the method of moments (Turchi et al. 1982, Benoit 1989, Benoit et al. 1992) (for lower momenta, the comparison with the method of moments cannot be done due to finite volume effects, Martin-Mayor et al. 2001). A very good agreement with the numerical data is achieved. Note the absence of the secondary peak in this model (see below).

We found that for densities down to $\rho \approx 0.6$, the agreement is good for all momenta, in fact good results are also obtained for the density of states (see figure (5])). For lower densities, the DOS starts to deviate from a Debye behavior at small $\lambda$, in contradiction with the numerical results. Moreover, below $\rho \approx 0.31$ the approximation gives a DOS with an unphysical negative spectrum. The negative spectrum develops continuously as a function of $\rho$. Also included in figure (5) is the one-loop result. Notice that even for $\rho=1$, the cactus resummation fails to reproduce 
the exponential decay of the density of states. This is not unexpected, however, due to the non perturbative nature (in $1 / \rho$ ) of this tail (Zee and Affleck, 2000).

It is interesting to look at the small $\lambda$ limit of $g_{\lambda}(\lambda)$. As seen in figure (5) (top) the behavior is very nearly Debye (i.e. $\sqrt{\lambda}$ ) for small $\lambda$. An excess of states relative to the Debye case develops for higher $\lambda$, but in a region of eigenvalues well beyond the linear dispersion regime (see bottom of figure (4)). Thus this peak, similar to that found in (Schirmacher et al. 1998), should not be regarded as a Boson peak.

Finally, let us look at the scaling of the position and the width of the peak in the frequency domain. In figure (6) we show the frequency corresponding to the Brillouin peak as a function of the external momentum $p$. Let us note that a nearly linear dependance, (pointing likely to a propagating excitation) persists up to $p / p_{0} \sim 0.6-0.7$. In figure $(7)$ instead we plot $\Gamma(p)$, obtained by means of (16). As expected, the $p^{2}$ scaling is found for very small momenta, which crosses over to a region where a simple law as (2) is not suitable to describe the real beahviour of the system.

Note that the region where the $p^{2}$ scaling is actually found, i.e. $p / p_{0}<0.1$ is quite different from the region explored by X-rays and neutrons scattering experiments, which rather spans the momenta $0.1<p / p_{0}<0.5$, It is worthwile to note that the same conclusion can be drawn from the results of Götze and Mayr using MCT for hard spheres (Götze \& Mayr 2000).

\section{CONCLUSIONS AND OUTLOOKS}

In summary, we have applied the random-matrix approach to the study of the high-frequency excitations of glassy systems. We have presented a resumation scheme (Grigera et al. 2001) that greatly enhances the predictive power of the $1 / \rho$ expansion prevoisuly obtained (Martin-Mayor et al. 2001). We have compared our analytical calculation with numerical results, finding that for not too low densities the only failure of the resumation scheme is its inability to repoduce the exponential decay of the density of states.

Our calculations have been performed choosing a gaussian force, in order to modelize the interparticle force $d r e s s e d$ with the pair correlation function, and only collinear displacements have been considered. We believe that our results are relevant for realistic glasses, at least in the regime $p / p_{0}<1$. The basic equation (17) shows that, in the general case, the position of the spectral peak is linear at small momenta, and the numerical solution of the integral equation in the Gaussian case shows that the linearity persists up to $p / p_{0} \sim 0.6-0.7$.

Moreover, the width of the spectral peak turns out to be proportional to $p^{2}$ in the limit of $p \rightarrow 0$ (see equation (18)) irrespective of the potential function $f(x)$. Interestingly, in the Gaussian case the scaling law holds for a momentum range one order of magnitude smaller than the experimental one. At larger momenta, the law is more complicated (see figure (7)). Being this result quite similar to MCT result for an hard-spheres system (Götze and Mayr 2000), we believe that it could have a certain degree of univerality.

This model lacks an important feature of the experimental spectra, namely the secondary peak of the $S(q, \omega)$ (which some authors have interpreted as the Boson peak, see Horbach et al. 1998). We believe this to be related to two important ingredients missing in our model: the vectorial nature of the vibration, and a detailed consideration of the particle correlations. The latter should not affect our results for momenta much smaller than the first maximum of the static structure factor, $p_{0}$. Since the secondary peak appears for $p \sim p_{0}$, we should not really expect to describe it: in this momentum range the dispersion relation is no longer monotonic in real glasses. Furthermore, Dell'Anna et al. 1998 have suggested that transversal excitations may play a prominent role in the raising of the secondary-peak. Our approach can be extended to include transverse displacements, though, and work in this direction is in progress.

VMM was partly supported by CICyT AEN99-0990, AEN97-1693 and M.E.C. TSG was supported in part by CONICET (Argentina).

\section{REFERENCES}

Allen P. B. ,Feldman J. L. Fabian J. and Wooten F. 1999 Phil. Mag. B 79

Angelani L. Di Leonardo R. Ruocco G. Scala A. Sciortino F. 2000 Phys. Rev. Lett. 85, 5356

Benassi P. Krisch M. Masciovecchio C. Mazzacurati V. Monaco G. Ruocco G. Sette F. and Verbeni R. 1996 Phys. Rev. Lett. 77, 3835

Benoit C. 1989 J. Phys.: Condens. Matter 1, 335

Biroli G. and Monasson R. 1999 J. Phys. A: Math. Gen. 32, L255

Buchenau U. Prager M. Ncker N. Dianoux A. J. Ahmad N. and Phillips W. A. 1986 Phys. Rev. B 34, 5665

Broderix K. Bhattacharya K. K. Cavagna A. Zippelius A. Giardina I. 2000 Phys. Rev. Lett. 85, 5360

Cavagna A. Giardina I. Parisi G. 1999 Phys. Rev. Lett. 83, 108 
Dell'Anna R. Ruocco G. Sampoli M. and Viliani G. 1998 Phys. Rev. Lett. 80, 1236

Ducastelle F. and Treglia G. 1982 J. Phys. C 15, 2891

Elliot S. R. 1983 Physics of amorphous materials (England: Longman)

Feldman J. L. Allen P. B. and Bickham S. R. 1999 Phys. Rev. B 59, 3551

Fioretto D. Buchenau U. Comez L. Sokolov A. Masciovecchio C. Mermet A. Ruocco G. Sette F. Willner L. Frick B. Richter D. and Verdini L. 1999 Phys. Rev. E 59, 4470

Foret M. Courtens E. Vacher R. Suck J. B. 1996 Phys. Rev. Lett. 77, 3831

Götze W. and Mayr M. R. 2000 Phys. Rev. E 61, 587

Grigera T.S., Martín-Mayor V., Parisi G. and Verrocchio P. 2001 cond-mat/0102230

Horbach J. Kob W. Binder K. 1998 J. Non-Cryst solids 235, 320

Horbach J. Kob W. Binder K. 1999 J. Phys. Chem. B 103, 4104

Kantelhardt J. W. Russ S. Bunde A. cond-mat/0012392; Phys. Rev. B (in press)

Keyes T. 1997 J. Chem. Phys. 101, 2921

Martín-Mayor V. Parisi G. Verrocchio P. 2000 Phys. Rev. E 62, 2373

Martín-Mayor V. Mèzard M. Parisi G. Verrocchio P. 2001 J. Chem. Phys. (in press)

Masciovecchio C. Ruocco G. Sette F. Krisch M. Verbeni R. Bergmann U. and Soltwisch M. 1996 Phys. Rev. Lett. $\mathbf{7 6}, 3356$

Masciovecchio C. Ruocco G. Sette F. Benassi P. Cunsolo A. Krisch M. Mazzacurati V. Mermet A. Monaco G. and Verbeni R. 1997 Phys. Rev. B 55, 8049

Masciovecchio C. Monaco G. Ruocco G. Sette F. Cunsolo A. Krisch M. Mermet A. Soltwisch M. and Verbeni R. 1998 Phys. Rev. Lett. 80, 544

Mazzacurati V., Ruocco G. and Sampoli M., 1996, Europhys. Lett. 34, 681

Metha M. L. 1991 Random matrices, (Academic Press)

Mèzard M. Parisi G. Zee A. 1999 Nucl. Phys. B559, 689

Monaco G. Masciovecchio C. Ruocco G. Sette F. 1998 Phys. Rev. Lett. 80, 2161

Montagna M. Ruocco G. Viliani G. Dell'Anna R. Di Leonardo R. Dusi R. Monaco G. Sampoli M. and Scopigno T., 1999 Phys. Rev. Lett. 83, 3450

Parisi G. 1994 Field-Theory, Disorder and Simulations, World Scientific.

Ribeiro M. C. C. Wilson M. and Madden P. A. 1998 J. Chem. Phys. 108, 9027

Ruocco G. Sette F. Di Leonardo R. Fioretto D. Krisch M. Lorenzen M. Masciovecchio C. Monaco G. Pignon F. and Scopigno T. 1999 Phys. Rev. Lett. 83, 5583

Ruocco G. Sette F. Di Leonardo R. Monaco G. Sampoli M. Scopigno T. and Viliani G. 2000 Phys. Rev. Lett. 84, 5788

Sampoli M. Benassi P. Dell'Anna R. Mazzacurati V. and Ruocco G. 1998, Phil. Mag. B 77, 473

Schirmacher W. Diezemann G. and Ganter C. 1998 Phys. Rev. Lett. 81, 136

Sette F. Krisch M. Masciovecchio C. Ruocco G. and Monaco G. 1998 Science 280, 1550

Sokolov A. P. Buchenau U. Richter D, Masciovecchio C. Sette F. Mermet A. Fioretto D. Ruocco G. Willner L. and Frick B. 1999 Phys. Rev. E 60, 2464

Taraskin S. N. and Elliot S. R. 1999 Phys. Rev. B 59, 8572

Taraskin S. N. Loh Y. L. Natarajan G. and Elliott S. R. 2001 Phys. Rev. Lett. 86, 1255

Turchi P. Benoit C. Royer E. and Poussigue G. 1992 J. Phys.: Condens. Matter 4, 3125

Wan Y. and Stratt R. 1994 J. Chem. Phys 100, 5123

Wu T. M. and Loring R. F. 1992 J. Chem. Phys. 97, 8368

Zee A. and Affleck I. 2000 J. phys: cond. mat. 12, 8863 


\section{ARGON}

\section{simulations}

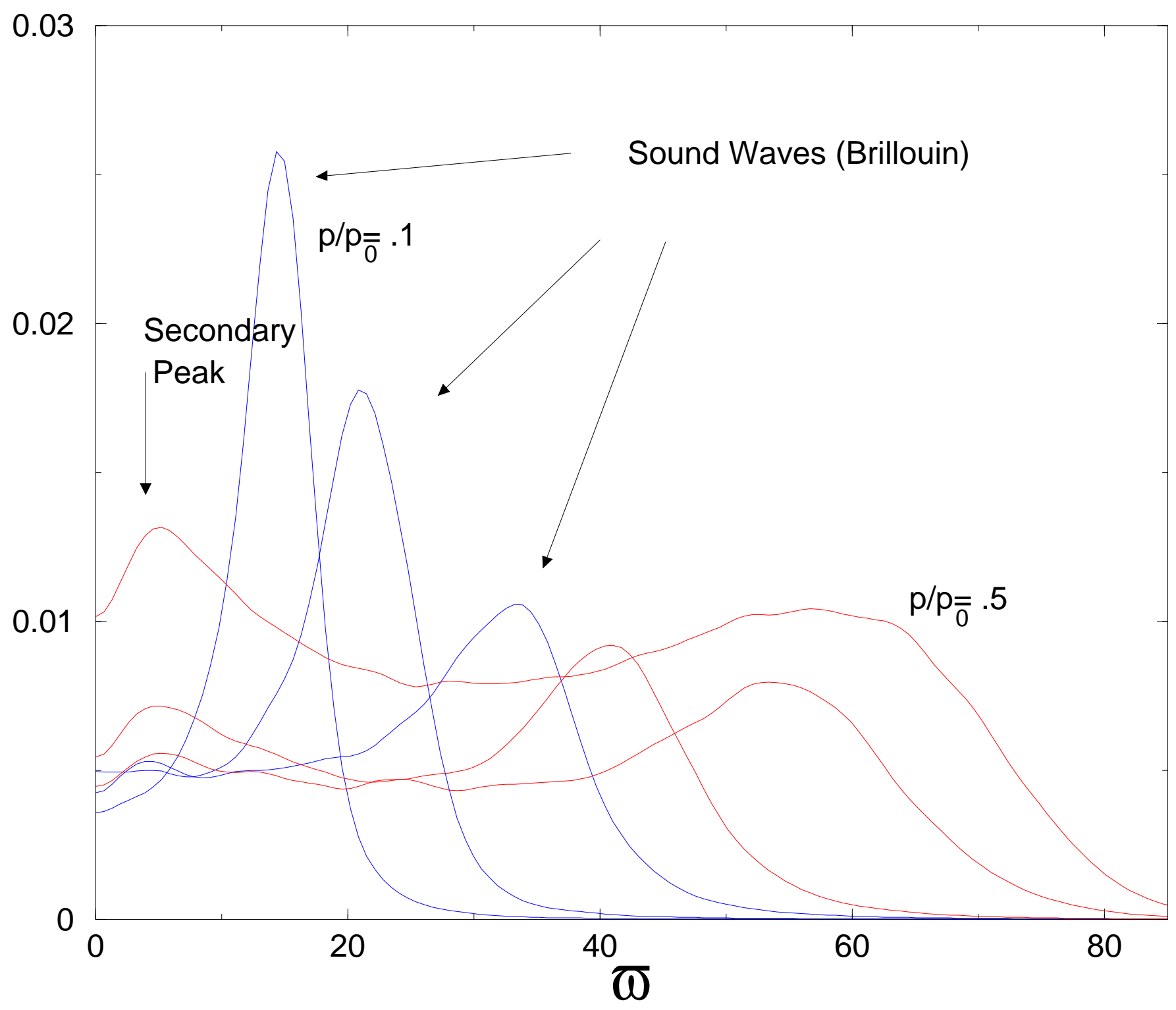

FIG. 1. Dynamical structure factor for argon, obtained numerically by Ruocco et al. 2000 at low temperature $\sim 10 K$. The Brillouin-like and the secondary peak appear quite clearly. 

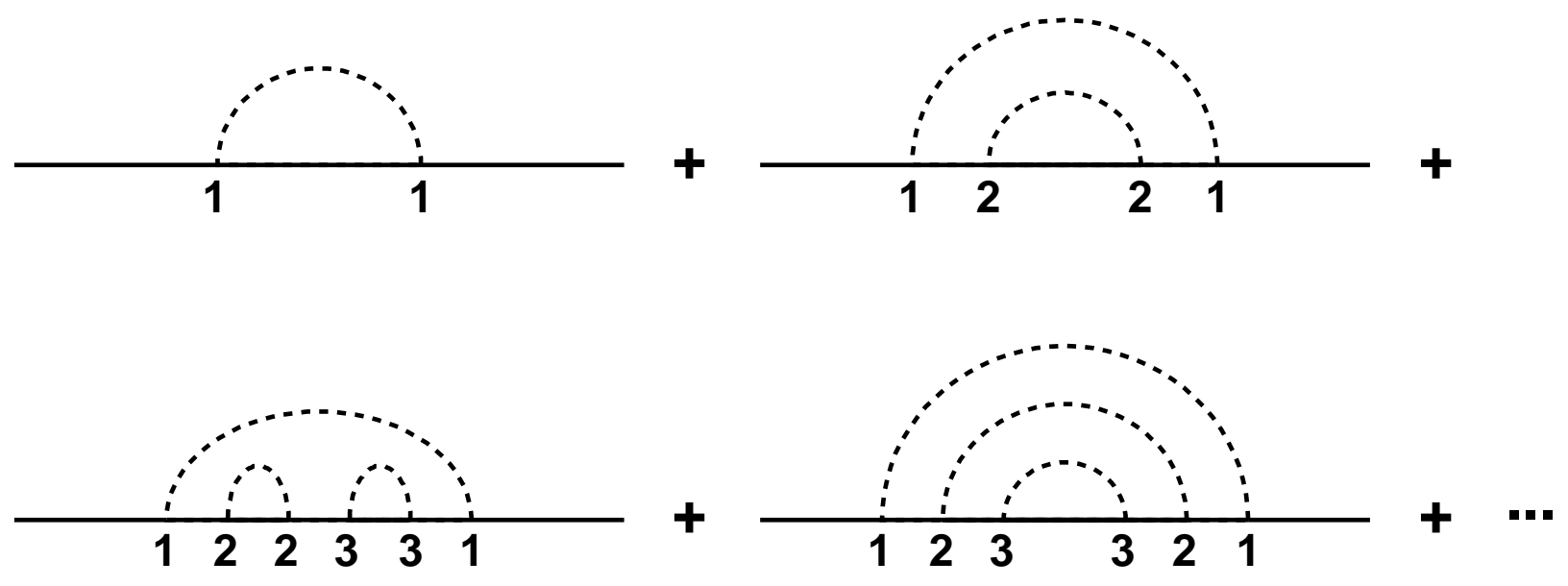

FIG. 2. Cactus diagrams of the $1 / \rho$ expansion. The numbers correspond to the particle-label repetitions 


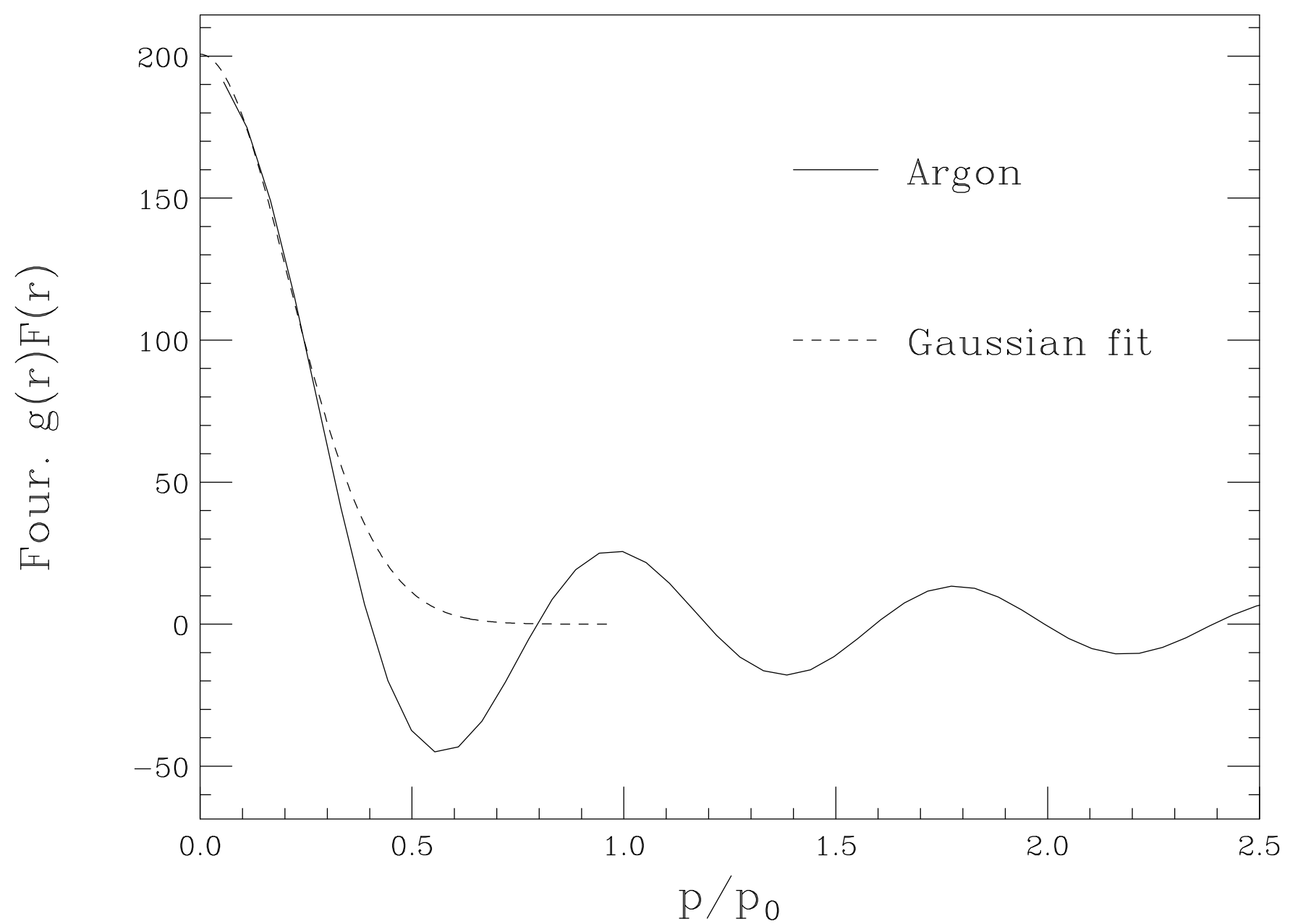

FIG. 3. The gaussian choice for $f(q)$ compared with the Fourier trasform of $g(r) F(r)$ at $\sim 10 K, F(r)$ being the second derivative of the Lennard-Jones pair potential which is supposed to modelize the Argon pair interactions. 


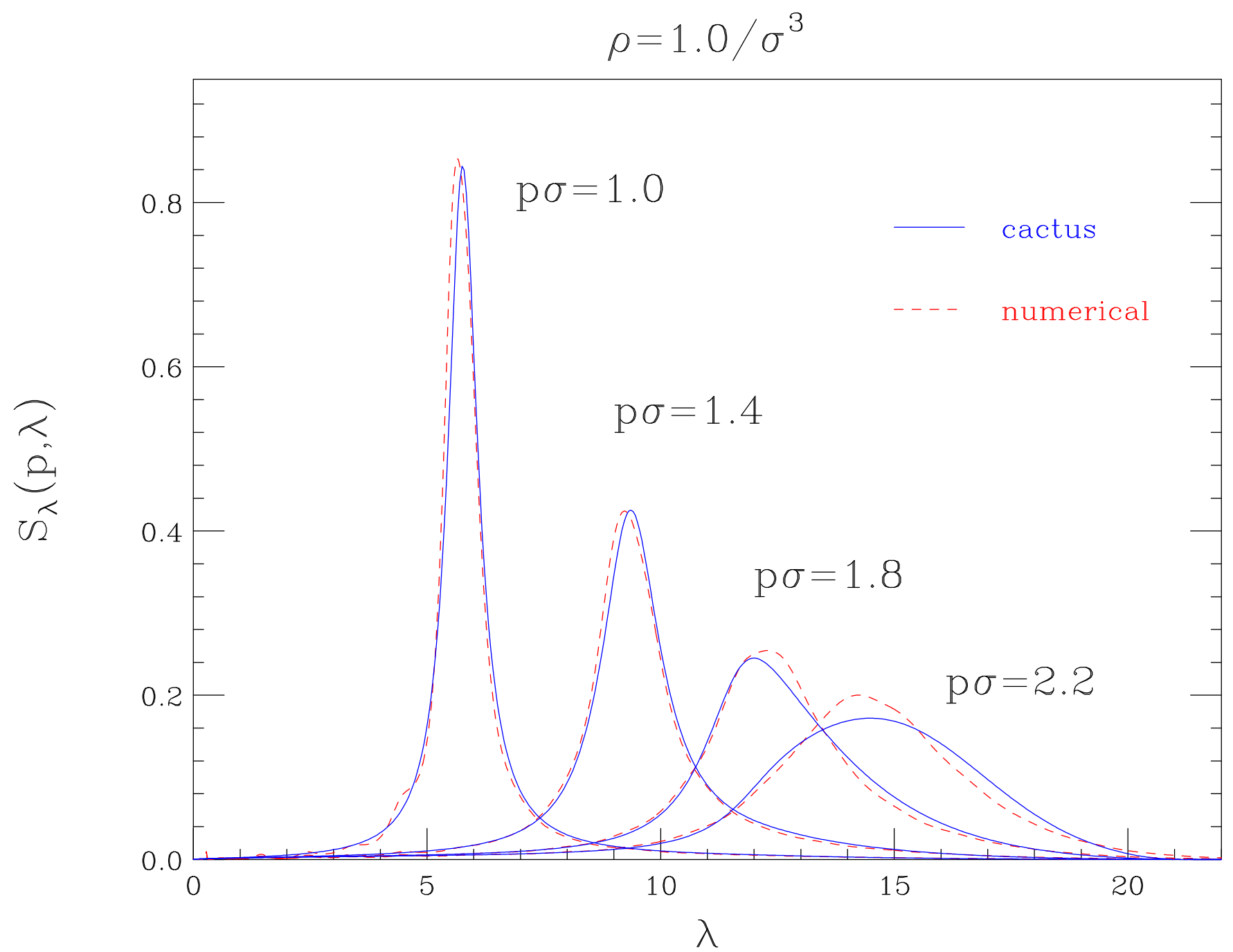

FIG. 4. Dynamical structure factor in the eigenvalues domain for several values of the momentum at $\rho=1$. 


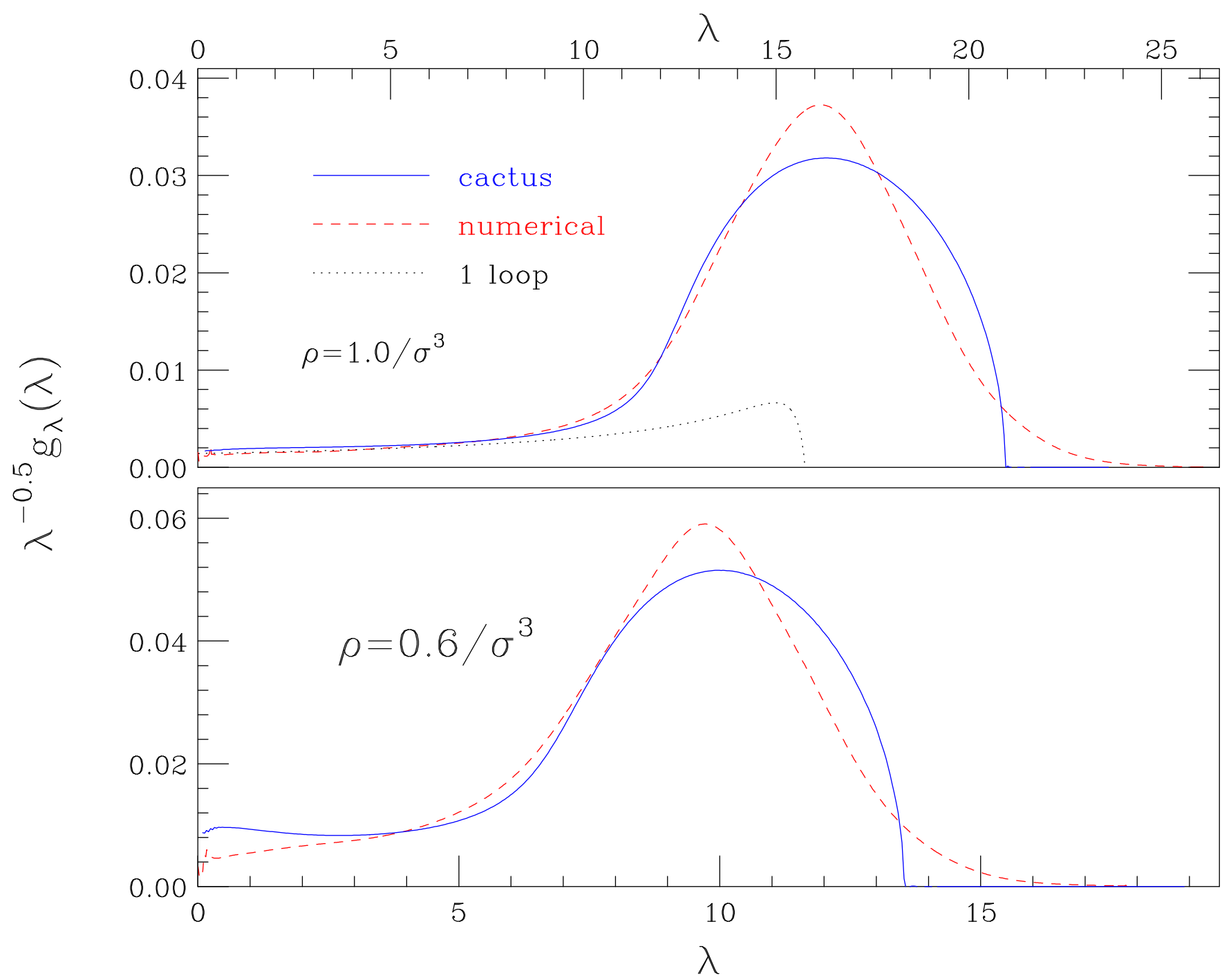

FIG. 5. Top: DOS in the eigenvalues domain divided by $\sqrt{\lambda}$ (Debye behavior) for $\rho=1$. Bottom: DOS for $\rho=0.6$. 


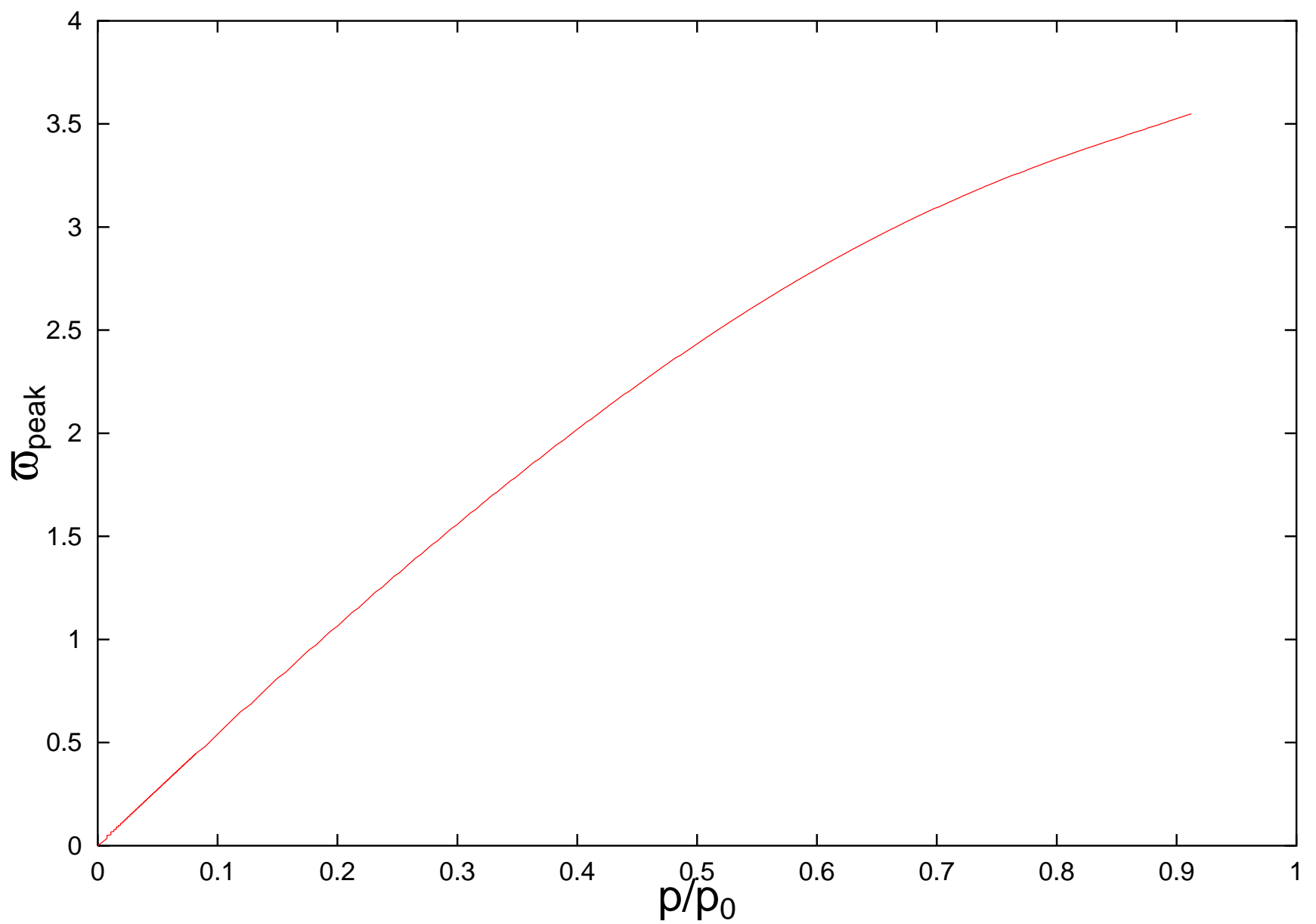

FIG. 6. Scaling behavior of the position of the peak, in the frequencies domain, for $r h o=1$. 


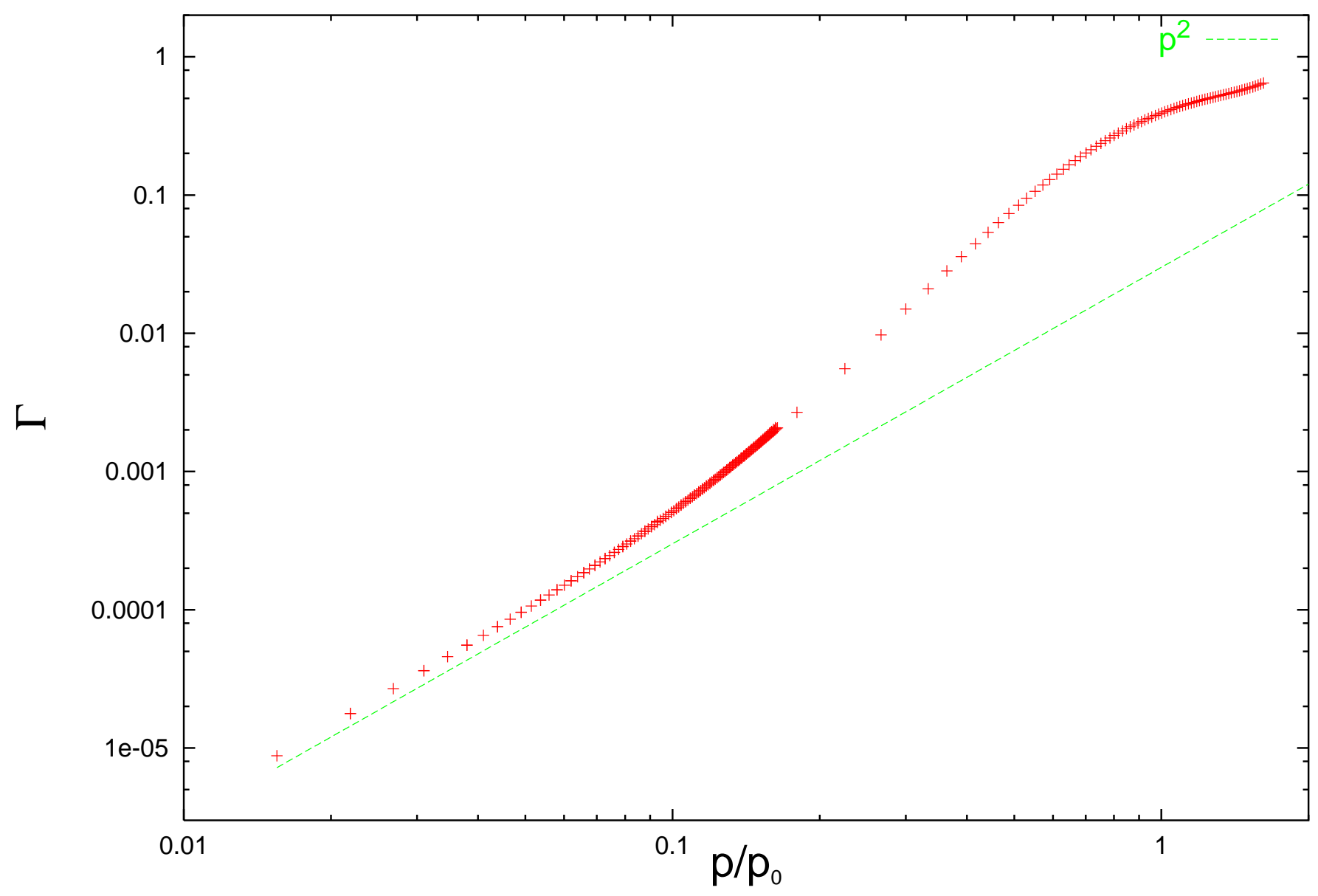

FIG. 7. Scaling behavior of the peak width at a function of $p$, in the frequencies domain, for $\rho=1$. 